\title{
A mechanical model for the prediction of the tire/road friction
}

\author{
M.-T. Do \& F. Hammoum \\ LCPC, Route de Bouaye, BP 4129, 44341 Bouguenais, France
}

Keywords: friction, microtexture, model, motif

\begin{abstract}
The viscoelastic deformation of tire rubber by road surface asperities was modeled by the rolling contact between a Kelvin solid and a surface motif, which is the part of surface profiles between two peaks. Stress and strain calculations showed that contact losses might occur locally, depending on the rolling speed, the viscoelastic properties of the Kelvin solid and the geometry of the motif. Mean vertical and horizontal forces were calculated, from which a coefficient of friction was derived. The model was successfully coupled with a profile analysis method to predict tire/road friction at low speed from road surface microtexture. The contribution of various texture scales to friction was emphasized.
\end{abstract}

\section{INTRODUCTION}

Skid resistance of pavements corresponds to their ability to generate friction forces between tire and road surface under conditions of braking, driving, cornering, free rolling or a composition of these modes. On dry road, friction forces are generally high. On wet road, the water film, which thickness may be only of a few ten microns, tends to separate the tire from the road and, by this fact, friction forces might be lowered compared with those obtained on dry surface. Skid resistance depends on various factors related to tire, environment and road surface characteristics. On slightly wetted surfaces, which is the most common situation, road surface microtexture is the dominant factor. Microtexture is defined as surface asperities less than $0.2 \mathrm{~mm}$-height and $0.5 \mathrm{~mm}$-width. They are required to breakdown the thin water film and restore contact condition between tire and road as close as possible to that encountered on dry surface. Values of tire/road friction depend also on deformation of tire rubber by microtexture asperities.

For highway engineers, it would be valuable to understand how friction is affected by surface microtexture, for they can act subsequently on this characteristic (e.g. by appropriate selection of aggregates) to enhance skid resistance. To this aim, model that relates microtexture to friction should be available. In addition, integration of such model into $\$ grand $\square$ models could enable prediction of friction at various speeds.

Few results were published on the relationship between microtexture and tire/road friction. Previous works reported mainly methods to characterize the geometry of microtexture asperities (Yandell 1971, Forster 1981, Himeno et al. 2000, Zahouani et al. 2000) and their correlation with friction. Yandell (1971) developed a numerical model to calculate stress and strain in rubber block flowing over triangular asperity, from which friction forces were derived. Measured friction was assumed to be the sum of friction forces generated at various texture scales, which could be calculated by means of the model. Comparison between prediction and measurement was reasonable (Yandell \& Sawyer 1994), but the model is quite complex to be of practical use.

In this paper, a contact model, called $\triangle$ Stefani model $\square$, was first developed, aiming to understand how friction force could be generated from contact between viscoelastic solid and surface asperity. The model was coupled with a profile analysis method in order to calculate friction from actual surface profiles. The ability of the model to predict low speed friction, where contribution of microtexture is dominant, was then investigated. 


\section{STEFANI MODEL}

\subsection{Model description}

The model geometry is related to pure rolling contact between Kelvin solid and surface motif (Fig. 1). The Kelvin solid is represented by a spring of modulus E connected to a dashpot of viscosity $\eta$. Motif is the part of surface profile between two consecutive peaks separated by a valley of $\mathrm{L}_{3^{-}}$width. The two peaks are characterized respectively by angles $\alpha_{1}$ and $\alpha_{2}$, widths $L_{1}$ and $L_{2}$, and angle $\theta_{12}$. Actually, $\theta_{12}$ is the angle between segment AD and the horizontal (Fig. 1). It is assumed that there is no surface friction during the rolling movement. Therefore, the model assumed that friction forces are generated uniquely by deformation of the Kelvin solid.

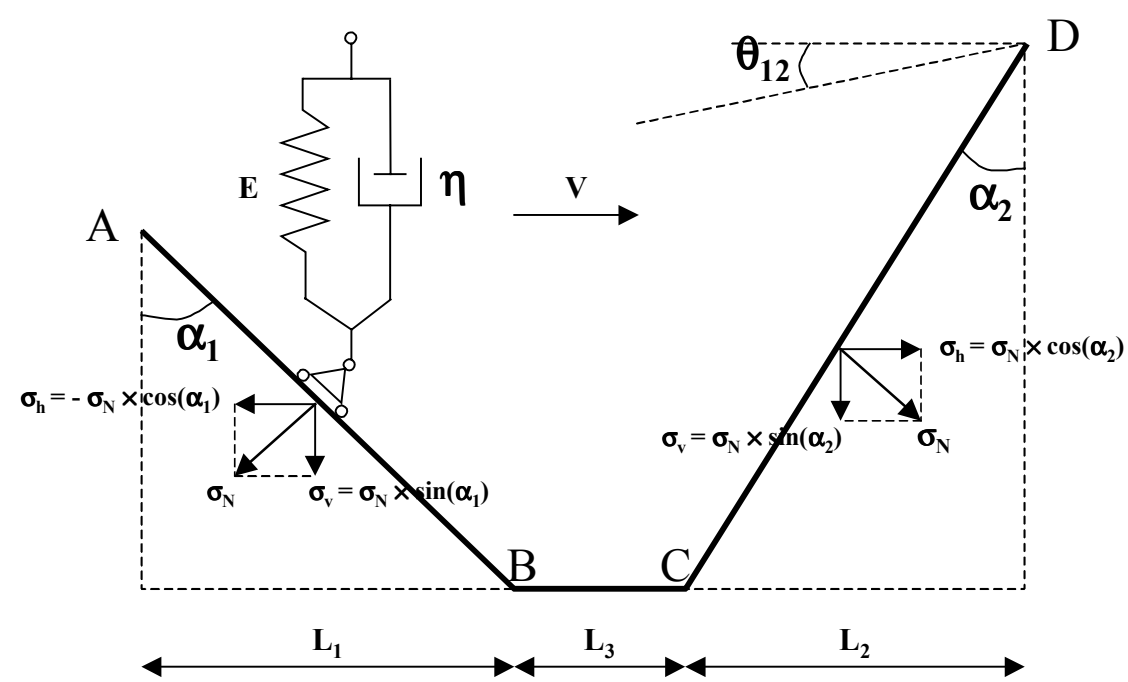

Figure 1. Geometry of the Stefani model.

The relationship between force $\sigma_{\mathrm{v}}$ and displacement $\varepsilon$ in Kelvin solid is:

$$
\sigma_{\mathrm{V}}=\mathrm{E} \varepsilon+\eta \frac{\partial \varepsilon}{\partial \mathrm{t}}
$$

On the AB segment, displacement at time $t$ is (Fig. 2):

$$
\varepsilon=\mathrm{h}_{0}+\mathrm{h}(\mathrm{t})=\mathrm{h}_{0}+\frac{\mathrm{L}_{1}-\mathrm{Vt}}{\tan \left(\alpha_{1}\right)}
$$

where $\mathrm{h}_{0}=$ initial displacement; and $\mathrm{V}=$ rolling speed.

Replacing $\varepsilon$ in (1) by (2) and deriving $\varepsilon$ with respect to time, we get

$$
\sigma_{\mathrm{V}}=\frac{\mathrm{E}}{\tan \left(\alpha_{1}\right)}\left[\mathrm{h}_{0} \tan \left(\alpha_{1}\right)+\mathrm{L}_{1}-\mathrm{V}(\mathrm{t}+\tau)\right]
$$

where $\tau=\eta / E=$ relaxation time of the Kelvin solid. 


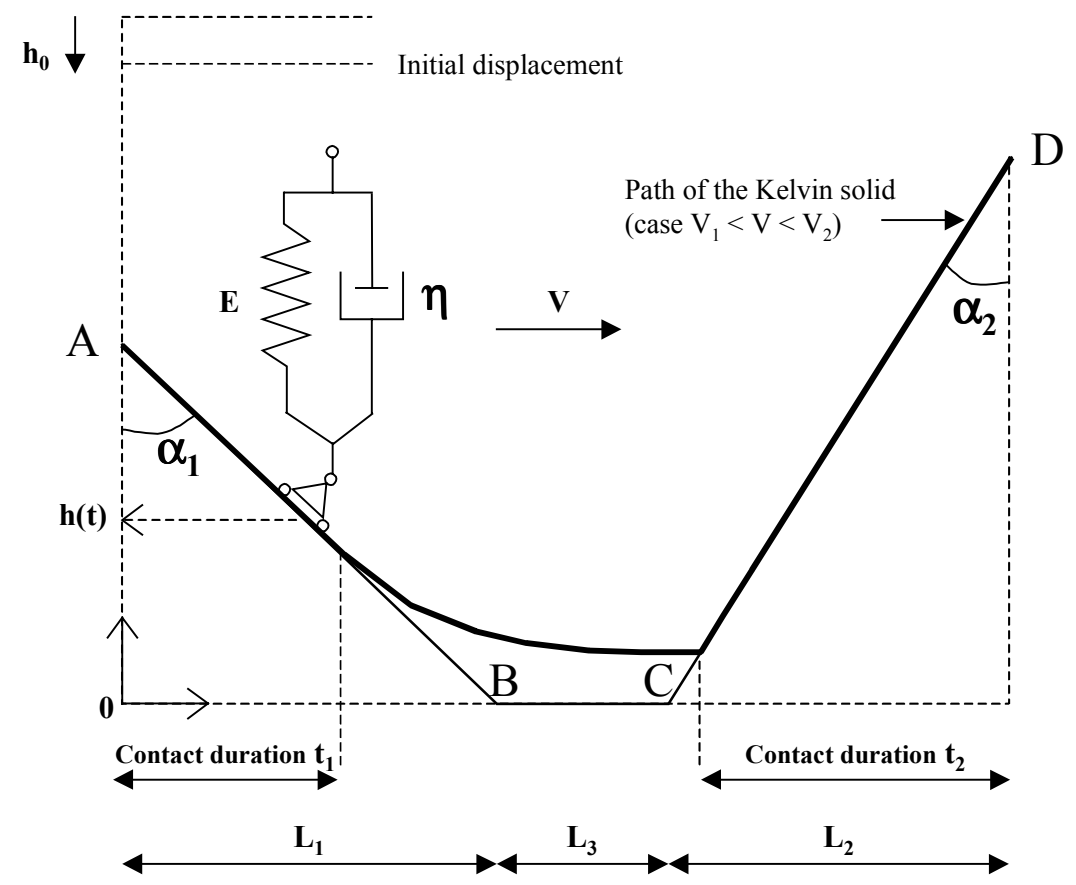

Figure 2. Kinematics of the Kelvin solid.

On the CD segment

$$
\sigma_{\mathrm{v}}=\frac{\mathrm{E}}{\tan \left(\alpha_{2}\right)}\left[\mathrm{h}_{0} \tan \left(\alpha_{2}\right)+\mathrm{V}(\mathrm{t}+\tau)-\mathrm{L}_{1}-\mathrm{L}_{3}\right]
$$

Sign examination of $\sigma_{\mathrm{v}}$ showed that contact loss $\left(\sigma_{\mathrm{v}}=0\right)$ might occur on AB with contact recovery on $\mathrm{BC}$ or $\mathrm{CD}$. Two critical speeds were defined (Stéfani, unpubl.):

$$
\mathrm{V}_{1}=\frac{\mathrm{h}_{0} \tan \left(\alpha_{1}\right)}{\tau}
$$

and

$$
\mathrm{V}_{2}=\frac{\mathrm{h}_{0} \tan \left(\alpha_{1}\right)+\mathrm{L}_{1}}{\tau}
$$

When $\mathrm{V}<\mathrm{V}_{1}$, no contact loss occurs between $\mathrm{A}$ and $\mathrm{D}$. When $\mathrm{V}_{1}<\mathrm{V}<\mathrm{V}_{2}$, contact loss occurs between $\mathrm{A}$ and $\mathrm{B}$. When $\mathrm{V}>\mathrm{V}_{2}$, contact loss occurs already at $\mathrm{A}$. Contact duration on $\mathrm{AB}$ and $\mathrm{CD}$ are respectively $t_{1}$ and $t_{2}$, defined as (Stéfani, unpubl.):

$$
\mathrm{t}_{1}=\frac{\mathrm{h}_{0} \tan \left(\alpha_{1}\right)+\mathrm{L}_{1}}{\mathrm{~V}}-\tau
$$

For $\mathrm{V}_{1}<\mathrm{V}<\mathrm{V}_{2}$

$$
\left[\frac{1}{\mathrm{e}} \frac{\mathrm{V}}{\mathrm{V}_{1}} \mathrm{e}^{\frac{\mathrm{V}_{1}-\mathrm{L}_{3} / \tau}{\mathrm{V}}}\right] \mathrm{e}^{-\frac{\mathrm{L}_{2} / \mathrm{V}-\mathrm{t}_{2}}{\tau}}=1+\frac{\mathrm{V}}{\mathrm{V}_{1}} \frac{\tan \left(\alpha_{1}\right)}{\tan \left(\alpha_{2}\right)} \frac{\mathrm{L}_{2} / \mathrm{V}-\mathrm{t}_{2}}{\tau}
$$


For $\mathrm{V}>\mathrm{V}_{2}$

$$
\left[\frac{\mathrm{V}_{2}}{\mathrm{~V}_{1}} \mathrm{e}^{\frac{\mathrm{V}_{1}-\mathrm{V}_{2}-\mathrm{L}_{3} / \tau}{\mathrm{V}}}\right] \mathrm{e}^{-\frac{\mathrm{L}_{2} / \mathrm{V}-\mathrm{t}_{2}}{\tau}}=1+\frac{\mathrm{V}}{\mathrm{V}_{1}} \frac{\tan \left(\alpha_{1}\right)}{\tan \left(\alpha_{2}\right)} \frac{\mathrm{L}_{2} / \mathrm{V}-\mathrm{t}_{2}}{\tau}
$$

\subsection{Calculation of the coefficient of friction}

Let us define $f_{v}$ and $f_{h}$ as respectively vertical and horizontal resultant forces from the rolling movement of the Kelvin solid over the motif. Calculation of $f_{v}\left(\right.$ respectively $f_{h}$ ) is obtained by integrating vertical (respectively horizontal) force at time $t, \sigma_{v}$ (respectively $\sigma_{h}$ ), over $t_{1}$ and $t_{2}$. For pure rolling contact (Fig. 1):

$$
\frac{\sigma_{\mathrm{h}}}{\sigma_{\mathrm{v}}}=\frac{1}{\tan \left(\alpha_{\mathrm{i}}\right)}
$$

where $\mathrm{i}=$ index taking values 1 and 2 respectively on $\mathrm{AB}$ and $\mathrm{CD}$.

Thus

$$
\begin{aligned}
& \mathrm{f}_{\mathrm{V}}=\frac{1}{\mathrm{~T}}\left\{\int_{0}^{\mathrm{t} 1} \frac{\mathrm{E}}{\tan \left(\alpha_{1}\right)}\left[\mathrm{h}_{0} \tan \left(\alpha_{1}\right)+\mathrm{L}_{1}-\mathrm{V}(\mathrm{t}+\tau)\right] \mathrm{dt}+\int_{0}^{\mathrm{t} 2} \frac{\mathrm{E}}{\tan \left(\alpha_{2}\right)}\left[\mathrm{h}_{0} \tan \left(\alpha_{2}\right)+\mathrm{L}_{2}-\mathrm{V}(\mathrm{t}-\tau)\right] \mathrm{dt}\right\}(10) \\
& \mathrm{f}_{\mathrm{h}}=\frac{1}{\mathrm{~T}}\left\{\int_{0}^{\mathrm{t}} 1-\frac{\mathrm{E}}{\left[\tan \left(\alpha_{1}\right)\right]^{2}}\left[\mathrm{~h}_{0} \tan \left(\alpha_{1}\right)+\mathrm{L}_{1}-\mathrm{V}(\mathrm{t}+\tau)\right] \mathrm{dt}+\int_{0}^{\mathrm{t} 2} \frac{\mathrm{E}}{\left[\tan \left(\alpha_{2}\right)\right]^{2}}\left[\mathrm{~h}_{0} \tan \left(\alpha_{2}\right)+\mathrm{L}_{2}-\mathrm{V}(\mathrm{t}-\tau)\right] \mathrm{dt}\right\}
\end{aligned}
$$

where $\mathrm{T}=\left(\mathrm{L}_{1}+\mathrm{L}_{2}+\mathrm{L}_{3}\right) / \mathrm{V}$.

The resultant coefficient of friction $\mu$ is given by

$$
\mu=\frac{f_{h}}{f_{v}}
$$

\subsection{Application of the model to road surface profiles}

Zahouani et al. (2000) developed a profile analysis method to characterize sharpness of microtexture asperities. On surface profiles, peaks and valleys are defined as points respectively higher and lower than their neighboring left and right points. Indenter is then defined as triangle composed of two valleys and the peak between the valleys (Fig. 3 ). Indenter summit angle $2 \alpha$ is given by

$$
\alpha=\frac{1}{2} \times\left[\tan ^{-1}\left|\frac{\mathrm{x}_{\mathrm{e}}-\mathrm{x}_{\mathrm{e}-1}}{\mathrm{z}_{\mathrm{e}}-\mathrm{z}_{\mathrm{e}-1}}\right|+\tan ^{-1}\left|\frac{\mathrm{x}_{\mathrm{e}+1}-\mathrm{x}_{\mathrm{e}}}{\mathrm{z}_{\mathrm{e}+1}-\mathrm{z}_{\mathrm{e}}}\right|\right]
$$

where $z_{e}=$ height of the $e^{\text {th }}$ extremum; and $x_{e}=$ abscissa of the $e^{\text {th }}$ extremum.

Relative position of profile peaks is characterized by angle $\theta$ defined as

$$
\theta=\tan ^{-1}\left|\frac{z_{p+1}-z_{p}}{x_{p+1}-x_{p}}\right|
$$

where $z_{p}=$ height of the $p^{\text {th }}$ peak; and $x_{p}=$ abscissa of the $p^{\text {th }}$ peak.

To be presented at the $6^{\text {th }}$ International Conference on the Bearing Capacity of Roads, Railways and Airfields (BCRA 02),

24-26 June 2002, Lisbon, Portugal. 


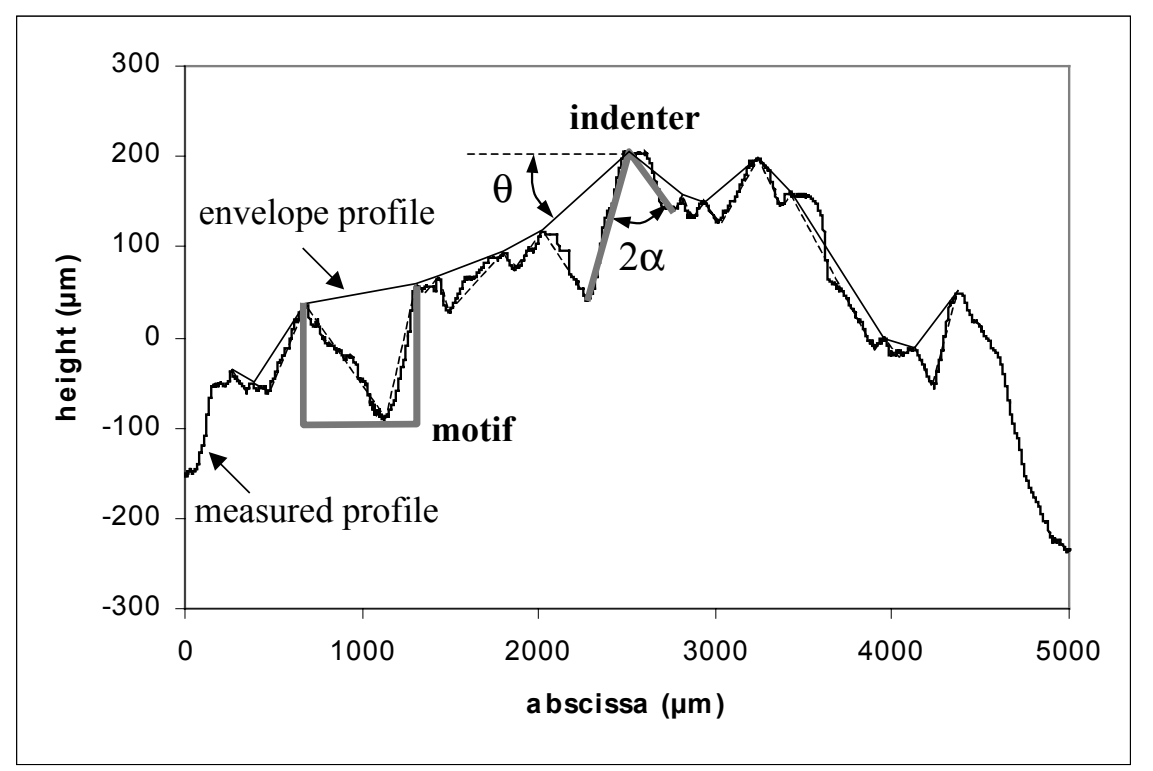

Figure 3. Illustration of indenter and motif.

It was found that cotangent $(\alpha)$ and $\theta$ are well correlated with friction measured by the Skid Resistance Tester (SRT friction) (Zahouani et al. 2000), which is representative of tire/road friction at low speed (Giles et al. 1964).

Actually, indenter and motif are quite equivalent, for they are defined from the same peaks and valleys population (Fig. 3). It was thought that the Stefani model might be coupled with the Zahouani et al. method to calculate tire/road friction from road surface profiles. Indeed, profile analyses provide mean values of cotangent $(\alpha), \theta$ and indenter width $2 \mathrm{~L}$. Assuming $\mathrm{L}_{3}=0$ and $\mathrm{L}_{1}=\mathrm{L}_{2}$, the mean surface motif could be characterized by

- cotangent $\left(\alpha_{1}\right)$, which is the mean value of cotangent $(\alpha)$

- angle $\theta_{12}$, which is the mean value of $\theta$

$-\mathrm{L}_{1}=\mathrm{L}_{2}=\mathrm{L}$

The angle $\alpha_{2}$ is given simply by

$$
\tan \left(\theta_{12}\right)=\frac{\mathrm{L}_{2} \cot \text { angent }\left(\alpha_{2}\right)-\mathrm{L}_{1} \cot \text { angent }\left(\alpha_{1}\right)}{\mathrm{L}_{1}+\mathrm{L}_{2}}=\frac{\cot \text { angent }\left(\alpha_{2}\right)-\cot \text { angent }\left(\alpha_{1}\right)}{2}
$$

Furthermore, assuming $\mathrm{h}_{0}=0$ and integrating (10) and (11), we get

$$
\mathrm{f}_{\mathrm{V}}=\frac{\mathrm{VE}}{2 \mathrm{~L}}\left[\frac{\mathrm{Lt}_{1}}{\tan \left(\alpha_{1}\right)}+\frac{\mathrm{Lt}_{2}}{\tan \left(\alpha_{2}\right)}-\frac{\mathrm{V}}{2}\left(\frac{\mathrm{t}_{1}^{2}}{\tan \left(\alpha_{1}\right)}+\frac{\mathrm{t}_{2}^{2}}{\tan \left(\alpha_{2}\right)}\right)-\mathrm{V} \tau\left(\frac{\mathrm{t}_{1}}{\tan \left(\alpha_{1}\right)}-\frac{\mathrm{t}_{2}}{\tan \left(\alpha_{2}\right)}\right)\right]
$$

and

$$
\mathrm{f}_{\mathrm{h}}=\frac{\mathrm{VE}}{2 \mathrm{~L}}\left[\frac{\mathrm{Lt}_{2}}{\left[\tan \left(\alpha_{2}\right)\right]^{2}}-\frac{\mathrm{Lt}_{1}}{\left[\tan \left(\alpha_{1}\right)\right]^{2}}-\frac{\mathrm{V}}{2}\left(\frac{\mathrm{t}_{2}^{2}}{\left[\tan \left(\alpha_{2}\right)\right]^{2}}-\frac{\mathrm{t}_{1}^{2}}{\left[\tan \left(\alpha_{1}\right)\right]^{2}}\right)+\mathrm{V} \tau\left(\frac{\mathrm{t}_{2}}{\left[\tan \left(\alpha_{2}\right)\right]^{2}}+\frac{\mathrm{t}_{1}}{\left[\tan \left(\alpha_{1}\right)\right]^{2}}\right)\right]
$$




\section{VALIDATION OF THE MODEL}

A validation program was performed in laboratory and results of which are presented in this paper. Ability of the model to predict tire/road friction at low speed from road surface microtexture is discussed. This is the first step before a larger validation program on roads.

\subsection{Experimental program}

\subsubsection{Specimens}

Rectangular specimens of $100 \mathrm{~mm} \times 150 \mathrm{~mm}$ were used. They are composed of 6 to10 $\mathrm{mm}$ coarse aggregates fixed in a resin matrix (Fig. 4). The procedure of fabrication of specimens was reported by Delalande (1992). Thirteen specimens were made, each being composed of aggregates from the same quarry. Due the procedure of fabrication, test surfaces are plane. This geometry emphasizes the contribution of surface microtexture to friction.

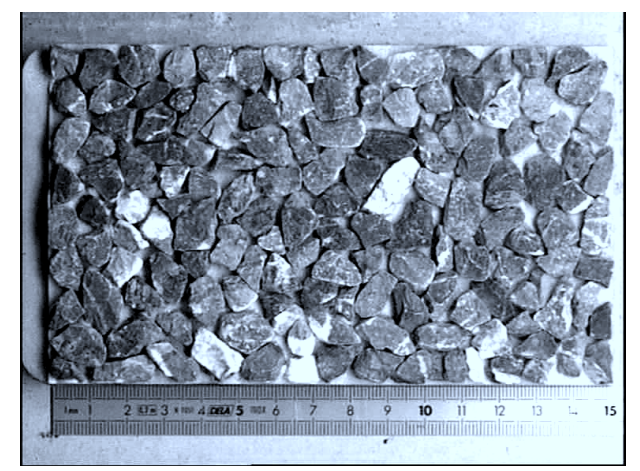

Figure 4. Example of specimen.

Specimens were subjected to polishing. Aggregates coming from stones with high polishing resistance preserve their microtexture. Aggregates coming from stones with low polishing resistance tend to smooth off. Microtexture difference between specimens is then emphasized by the polishing action. The polishing device is called $\backslash$ GRAP $\square$, which was developed in France by the LPC network (Public Works Regional Laboratories) as an alternative to the British Accelerated Polishing Test (Delalande 1992). The polishing action is achieved by the projection of a water and very fine abrasive mix under a pressure of $10 \mathrm{MPa}$ by means of a nozzle with a $60^{\circ}$-incidence angle (Fig. 5). The surface is swept by the displacements of the projection nozzle. Twenty sweep cycles are necessary to obtain the GRAP limit polishing state. The degree of polishing is assessed by the SRT friction, which is measured under the same test conditions as those used for road surfaces. The test method is detailed in (Delalande 1992). Results from the same reference showed that the limit polishing states of the GRAP and British Accelerated Polishing Test methods are quite comparable. 


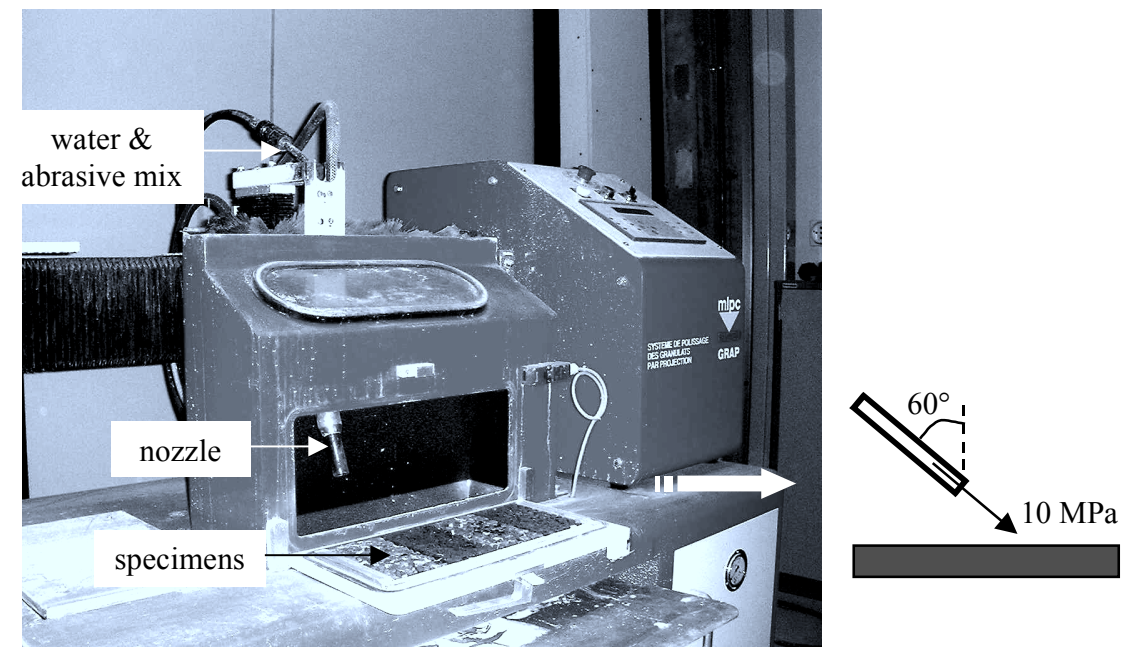

Figure 5. Polishing device.

\subsubsection{Friction measurements}

Friction was measured by means of a Skid Resistance Tester (SRT) (Fig. 6). This device is widely used in order to assess the skid resistance properties at low speed of a surface either in the field or in the laboratory. The tester incorporates a spring-loaded $76.2 \mathrm{~mm} \times 25.4 \mathrm{~mm}$ slider made of a standard rubber attached to the end of a pendulum. On releasing the pendulum from a horizontal position, the loss of energy as the slider passes over the test surface is measured by the reduction in length of the upswing using a calibrated scale.

The surfaces were wetted before release of the slider. Measurements were done in laboratory where ambient temperature was kept constant $\left(20^{\circ} \mathrm{C}\right)$. Thirteen releases were made, the last three readings being taken for calculating the SRT friction values.

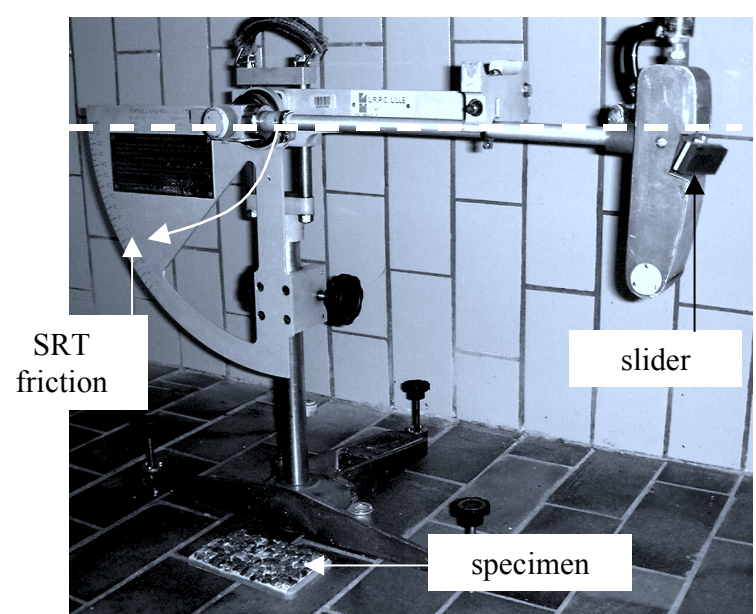

Figure 6. Skid Resistance Tester device.

\subsubsection{Microtexture measurements}

Microtexture profiles were measured by means of a tactile sensor. The radius of the contact cone is of $2 \mu \mathrm{m}$. The measuring range is of $6 \mathrm{~mm}$. On each specimen, 15 profiles were measured in the friction area of the SRT $(75 \mathrm{~mm} \times 125 \mathrm{~mm})$. Profile lengths varied between 12 and $25 \mathrm{~mm}$, depending on the topography of the measured surface. Cumulative lengths were about $300 \mathrm{~mm}$ for each specimen. Sampling interval was of $4 \mu \mathrm{m}$.

To be presented at the $6^{\text {th }}$ International Conference on the Bearing Capacity of Roads, Railways and Airfields (BCRA 02),

24-26 June 2002, Lisbon, Portugal. 


\subsection{Results}

\subsubsection{Model input}

Beside profile data, the following input is required for the calculations:

- rolling speed $\mathrm{V}$

- relaxation time of the Kelvin solid

- critical speeds $\mathrm{V}_{1}(5)$ and $\mathrm{V}_{2}(6)$

- contact duration $\mathrm{t}_{1}(7)$ and $\mathrm{t}_{2}(8)$ or (8bis)

The rolling speed was assumed to be the speed of SRT rubber slider when it passes over the test surface, which is of $3 \mathrm{~m} / \mathrm{s}$. For $\mathrm{V}_{1}=0$ (assuming $\mathrm{h}_{0}=0$ ), there is local loss of contact. The relaxation time was assumed to be $\tau=10^{-6} \mathrm{~s}$. Thus $\mathrm{V}_{2}=\mathrm{L} / \tau=10^{6} \mathrm{~L}$. The rolling speed $\mathrm{V}$ would be then less than $\mathrm{V}_{2}$ if $\mathrm{L}>3 \mu \mathrm{m}$. For the profile sampling interval is of $4 \mu \mathrm{m}$, the minimum value of $\mathrm{L}$ will be of $4 \mu \mathrm{m}$. Therefore, we always have $\mathrm{V}_{1}<\mathrm{V}<\mathrm{V}_{2}$.

The duration $\mathrm{t}_{2}$ is obtained by solving the equation

$$
\mathrm{Te}^{(1+\mathrm{T})}=\frac{\tan \left(\alpha_{2}\right)}{\tan \left(\alpha_{1}\right)}
$$

where $\mathrm{T}=\frac{\mathrm{L} / \mathrm{V}-\mathrm{t}_{2}}{\tau}$.

Values of $\mathrm{T}$ for this limited experimental program are almost equal to 0.13 . This value was then used for the 13 specimens to calculate $t_{2}$.

\subsubsection{Comparison between measured and calculated frictions}

From microtexture profiles measured on each specimen, calculations by the Stefani model require the following steps:

- analysis of the profiles by the Zahouani et al. method: calculation of cotangent $(\alpha), \theta$ and L for each indenter; values from all the profiles are then regrouped from which respective mean values are calculated

- definition of the mean motif for each specimen, using the correspondence given in 2.3

- calculation of $t_{1}$ from (7) and $t_{2}$ from (18)

- calculation of $f_{v}$ from (16) and $f_{h}$ from (17), then $\mu$

Comparison between friction calculated by the Stefani model and SRT friction is presented in the Figure 7 ( $\square$ white $\square$ circles). Prediction is lower than observation, the difference being larger for high friction surfaces. However, close relationship is obtained between measurement and calculation, confirming the ability of the Stefani model to calculate friction from actual surface profiles.

It was thought that prediction from the model could be improved by taking into account the contribution of other texture scales. The question is how separation of texture scales on surface profiles should be done. Various filtering techniques were proposed in the past. Yandell and Sawyer (1994) for example used a $5^{\text {th }}$ order Bessel filter to divide profile into 4 bands. The motif combination technique, from which the method of Zahouani et al. was derived, divides profile into two scales (Fahl 1982) (Fig. 3):

- roughness scale related to the measured profile

- undulation scale related to the envelope profile, which is composed of segments connecting peaks of the measured profile

Definition of these two scales was motivated by the fact that profile and its envelope could have distinct functional properties. In this paper, the filtering technique based on motif combination was chosen for its simplicity and for the fact that surface motif concept is used in the Stefani model. Calculations should be then performed on two mean motifs characterizing respectively the measTo be presented at the $6^{\text {th }}$ International Conference on the Bearing Capacity of Roads, Railways and Airfields (BCRA 02), 24-26 June 2002, Lisbon, Portugal. 
ured profiles and their envelopes. Resultant coefficients of friction are expressed respectively as $\mu_{\text {rough }}$ and $\mu_{\text {undul }}$, and it might be assumed that $\mu=\mu_{\text {rough }}+\mu_{\text {undul. }}$. It could be stated that the first comparison presented by the $\square$ white $\square$ circles took into account only the contribution of the roughness scale $\mu_{\text {rough }}$. The comparison between SRT friction and the sum $\left(\mu_{\text {rough }}+\mu_{\text {undul }}\right)$ are presented by $\square$ black $\square$ circles in Figure 7. Points are now close to the bisecting line. These results confirm the assumed contribution of the roughness and undulation scales.

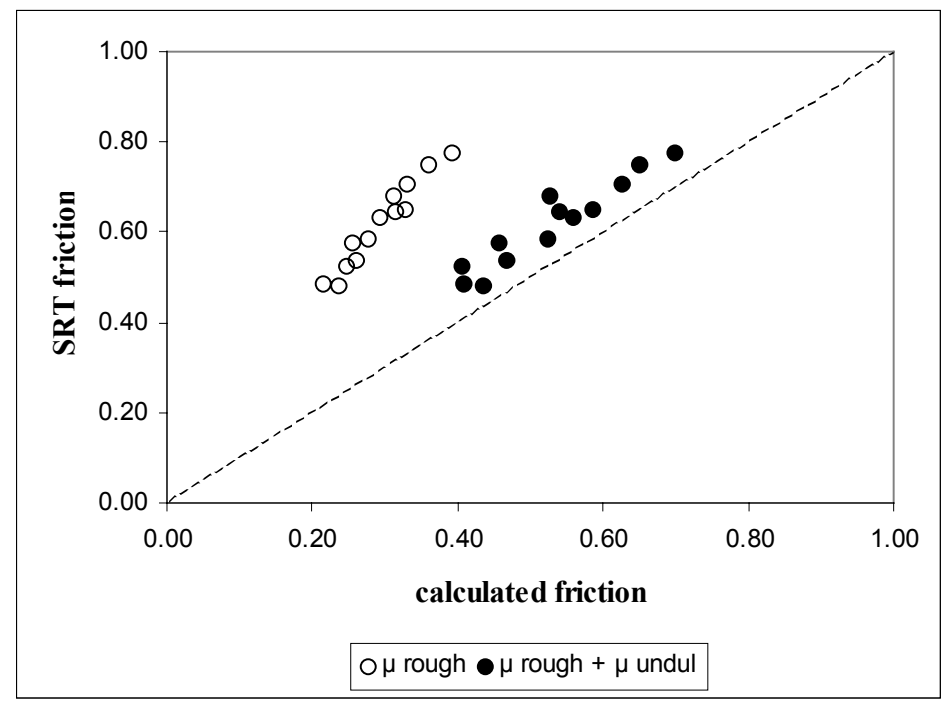

Figure 7. Comparison between calculation and measurement.

\section{CONCLUSIONS}

In this paper, a contact model was developed to see how friction forces could be generated from contact between viscoelastic solid and rough surface. In order to keep the model simple, pure rolling contact between Kelvin solid and surface motif was described. Motif is the part of surface profile between two peaks and could be defined by simple geometric considerations. The model was later coupled with a profile analysis method to calculate tire/road friction from actual profiles of surface texture. Friction calculation require only characteristics of the mean motif characterizing surface texture, relaxation time and rolling speed of Kelvin solid.

Experimental program was developed in laboratory to validate the ability of the model to predict low speed friction from surface microtexture. Test surfaces are composed of aggregates to enhance the contribution of surface microtexture. Standard Skid Resistance Tester device was used, for resulting friction values are representative of skid resistance at low speed of roads. Results showed that the model predict reasonably well observed friction. Calculations must take into account the contribution of two microtexture scales, defined simply from measured profiles and their envelopes.

The model needs further validation, mainly by tests on actual roads. Prediction of tire/road friction at various speeds must also be investigated. However, the first results are promising and, by the simple concepts employed, the model could be a practical tool for highway engineers to assess the relationship between microtexture and tire/road friction. 


\section{ACKNOWLEDGEMENT}

The authors thank M. Christian Stéfani, researcher at LCPC, for the development of the contact model and his advice during the validation phase.

\section{REFERENCES}

Delalande, G. 1992. The Resistance of Aggregates to Polishing: A Projection Test (in French). Bulletin de Liaison des Laboratoires des Ponts et Chaussées 177: 73-80.

Fahl, C. F. 1982. Motif Combination $\square$ A New Approach to Surface Profile Analysis. Wear 83: 165-179.

Forster, S. W. 1981. Aggregate Microtexture : Profile Measurement and Related Friction Levels. Report FHWA/RD-81/107. FHWA, US Department of Transportation.

Giles, C. G., Sabey, B. E. \& Cardew, K. H. F. 1964. Development and Performance of the Portable SkidResistance Tester. Road Research Technical Paper 66. Road Research Laboratory, Department of Scientific and Industrial Research.

Himeno, K., Nakamura, Y., Kawamura, A. \& Saito, K. 2000. Skid Resistance of Asphalt Pavement Surfaces Related to their Microtexture. Pavement Surface Characteristics of Roads and Airfields; Proceedings of the 4th International Symposium, Nantes, France, 22-24 May 2000. Paris: PIARC.

Yandell, W. O. 1971. A New Theory of Hysteretic Sliding Friction. Wear 17: 229-244.

Yandell, W. O. \& Sawyer, S. 1994. Prediction of Tire-Road Friction from Texture Measurements. Transportation Research Record 1435: 86-91.

Zahouani, H., Vargiolu, R. \& Do, M.-T. 2000. Characterization of Microtexture Related to Wet Road/Tire Friction. Pavement Surface Characteristics of Roads and Airfields; Proceedings of the 4th International Symposium, Nantes, France, 22-24 May 2000. Paris: PIARC. 